\title{
Localización espacial del uso del suelo poblacional del municipio de San José Colinas, Santa Bárbara, (Honduras), uti- lizando como herramienta un sistema de información geográfico
}

Yeny Castellanos

\section{Resumen}

Durante los últimos años, el Municipio de San José Colinas, Santa Bárbara presenta un aumento de solicitudes de ejecución de planes y proyectos para el municipio en todas sus áreas. Razón por la cual se necesita identificar que las características geográficas y demográficas del municipio (edad, sexo, contexto familiar)necesarias o faltantes en los datos geoespaciales que equilibren la implementación de los servicios básicos como parte de la satisfacción de las necesidades humanas y es en donde con la ayuda de los método y técnicas de los Sistemas de Información Geográfica (SIG) será un aporte en los procesos de mejora para el desarrollo del municipio.

Cuando se habla de las desigualdades de los servicios básicos son problemas de atención primaria, que se deberían minimizar por las autoridades locales.

Razón por la cual es necesario el desarrollo de la metodología en diversas fases en donde se identificaron las zonas de las áreas de estudio del Municipio de San José Colinas, Santa Bárbara, con la colaboración de la Dirección de Vinculación Universidad Sociedad. Encontrándose con el $60 \%$ de los datos tabulados hasta la fecha.

Palabras clave: Servicios básicos, métodos, técnicas, sistema de información geográfica

\section{Abstract}

In recent years, the Municipality of San Jose Hills, Santa Barbara presents an increase of requests for implementation of plans and projects for the city in all its areas. Why you need to identify the geographic and demographic characteristics 
of the municipality (age, sex, family context) necessary or missing geospatial data to balance the implementation of basic services as part of the fulfillment of human needs and is in where with the help of the method and techniques of Geographic Information Systems (GIS) will be a contribution to the improvement processes for the development of the municipality.

When we talk about inequality of basic services they are primary care problems, which should be minimized by local authorities.

Why the development of the methodology at various stages in the areas where the study areas of the Municipality of San Jose Hills, Santa Barbara, in collaboration with the Directorate for University Society identified need. Encountering $60 \%$ of the tabulated data to date.

Key words: Basic services, methods, techniques, GIS

Yeny Castellanos, Departamento de Ciencia y Tecnologías de la Información Geográfica -

Facultad de Ciencias Espaciales,Universidad Nacional Autónoma de Honduras 


\section{Introducción}

Los servicios básicos encierran una cantidad de actividades desarrolladas por el ser humano, para la satisfacción de las necesidades que cada vez son de vital importancia cubrir, son necesarios identificar estos servicios básicos como equipamientos físicos o como estructuras activas, aplicando las tecnologías de la información geográfica se incluyen patrones espaciales de ubicación, cantidad y características que dan a la población oportunidades desiguales de uso y disfrute de estos servicios. En donde la accesibilidad de una población que posea estos servicios lleva a cubrir estas necesidades básicas y al mismo tiempo dar seguridad y bienestar.

Geográficamente este punto adquiere una trascendencia singular, por cuanto está bien demostrado hasta qué punto tal uso y satisfacción depende de factores espaciales como la distancia entre el destinatario y el punto de oferta del servicio.

Las definiciones teóricas que hacen referencia a la accesibilidad y sus correspondientes expresiones matemáticas han sido llevadas también al ámbito geométrico, ello ha permitido desarrollar modelos de la realidad a partir de los cuales se pueden establecer áreas con accesibilidad diferencial en un territorio determinado. Estos modelos pueden ser fácilmente incorporados a los Sistemas de Información Geográfica que se han convertido en las últimas décadas herramientas idóneas para evaluar y valorar la accesibilidad de la población a determinados bienes y servicios.(Ramírez, 2003)

\section{Datos y Metodología}

Se considera la organización de las tareas en fases como una guía que se adapte a las particularidades de la investigación.

Como guía metodológica se ha tomado del estudio desarrollado por Antonio Moreno(Moreno, 2008), el cual fue desarrollado en fases que se describen a continuación:

FASE I: Diagnóstico de la situación. En la fase de diagnóstico de la situación se trata de determinar los problemas actualmente existentes en el sistema de provisión y uso de los servicios colectivos. 
Elementos típicos a abordar en ella conciernen a:

- Identificación de zonas de estudio

- Identificación de inventario de los equipamientos, de los recursos disponibles y de sus atributos.

- Base de datos con las delimitaciones de las áreas de servicio actuales y sus atributos, zonas escolares, áreas de salud, áreas de servicios sociales, etc.

- Base de datos espacial de los usuarios actuales y sus rasgos relevantes.

- Base de datos espacial de la población o de los segmentos de la demanda objetivo.

- Bases de geodatos digitales relativas a las infraestructuras y medios para el transporte (vías -calles, carreteras, etc.-, líneas de transporte, paradas y estaciones, etc.), y plano parcelario.

- Bases de datos espaciales de actividades, instalaciones y usos del suelo relevantes (especialmente los que interactúan con los equipamientos en cuestión y con su funcionamiento regular).

FASE II: Estudio de necesidades y dimensionamiento de la demanda espacial.

- Examen de indicadores de logros y satisfacción-insatisfacción de los usuarios o afectados. Habitualmente los datos se referirán a unidades zonales relevantes, o bien a cada uno de los centros de servicio.

- Análisis de la accesibilidad espacial actual a los puntos de servicios desde cada lugar con demanda.

- Caracterización de las pautas de conducta espacial de los usuarios o de distribución del servicio a domicilio por parte del proveedor.

- Determinación de las incompatibilidades entre los equipamientos y otros usos del suelo, derivados de una proximidad mutua excesiva.

- Visualización exploratoria de datos y presentación cartográfica de resultados.

- Búsquedas temáticas, espaciales y mixtas.(Hein, 2009) 
FASE III: Formulación de esquemas de distribución de los equipamientos y funcionamiento espacial de los servicios.

Geográficamente una faceta obligada en esta fase consiste en obtener una desagregación espacial de la misma, esto es, conocer a cuántos y quiénes habría que atender en cada lugar. Dependiendo de los objetivos avistados en la planificación, puede ser necesario considerar no tanto el escenario actual, sino otro futuro, o varios momentos a lo largo de un período posterior.

FASE IV: Decisiones. A partir de los hallazgos es posible ya percibir la tarea de definir qué patrón espacial de la oferta, de dotaciones, y qué forma de funcionamiento espacial de los servicios provistos resulta más apropiada para resolver los problemas diagnosticados y satisfacer la demanda identificada. Aquí típicamente resultan apropiadas técnicas como los modelos de localización óptima.

FASE V: Evaluación de logros. El objetivo de la evaluación recae en determinar si las decisiones adoptadas están alcanzando los fines avistados o si deben ser revisadas en algunos de sus aspectos.

\section{Resultados}

- Se han identificado zonas de áreas de estudio del Municipio de San José Colinas, Santa Bárbara, con la colaboración de la Dirección de Vinculación Universidad Sociedad. (Cuadro 1).

El mapa de localización muestra la ubicación geográfica del Municipio de San José Colinas el cual es la zona de estudio con la distribución de todas sus aldeas (Figura 1). 


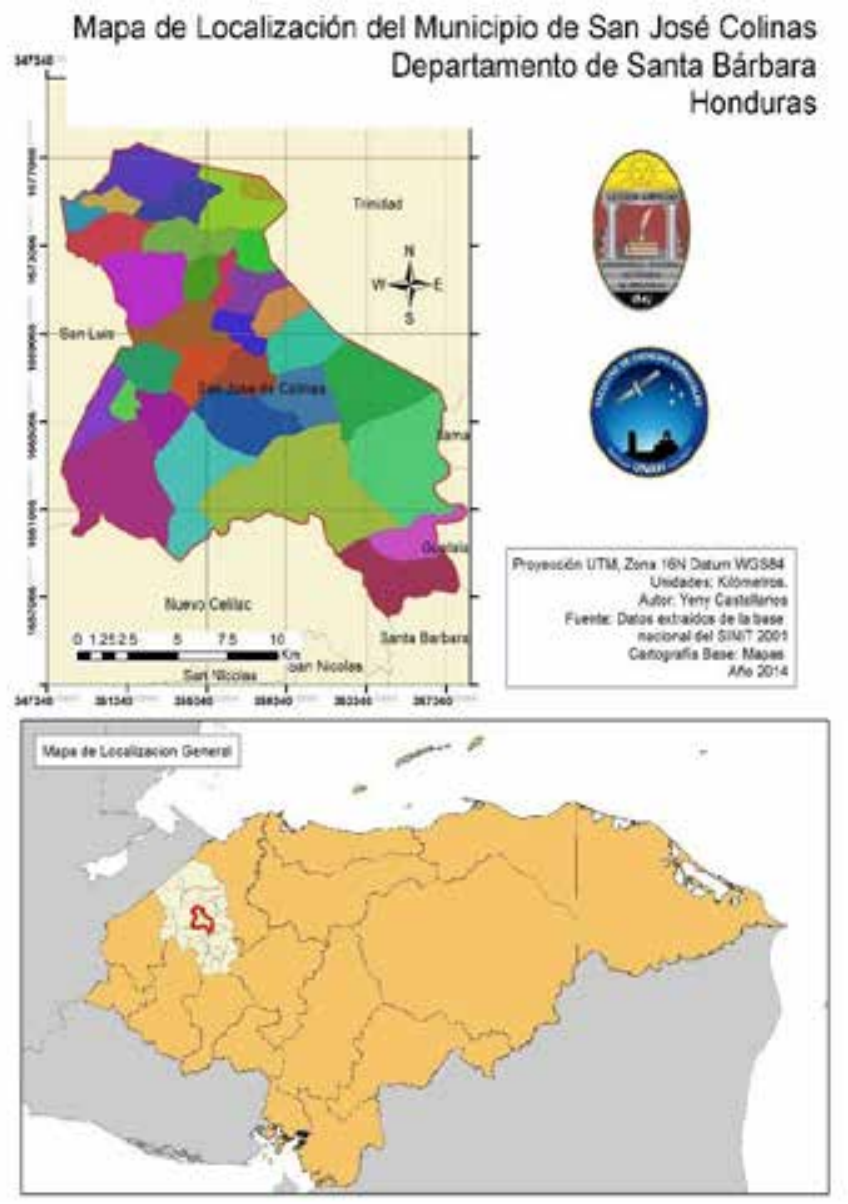

La relación de la distribución de la población respecto a la hidrografía de la zona se puede observar en el mapa de Hidrografía e infraestructuras, esta última se desarrollaron en las zonas pobladas de todo el municipio lo cual se puede observar parte de la vulnerabilidad en tiempos de lluvias (Figura 2). 
Hidrografia e Infraestructura de Aldeas San Jose Colinas

Departamento de Santa Bárbara

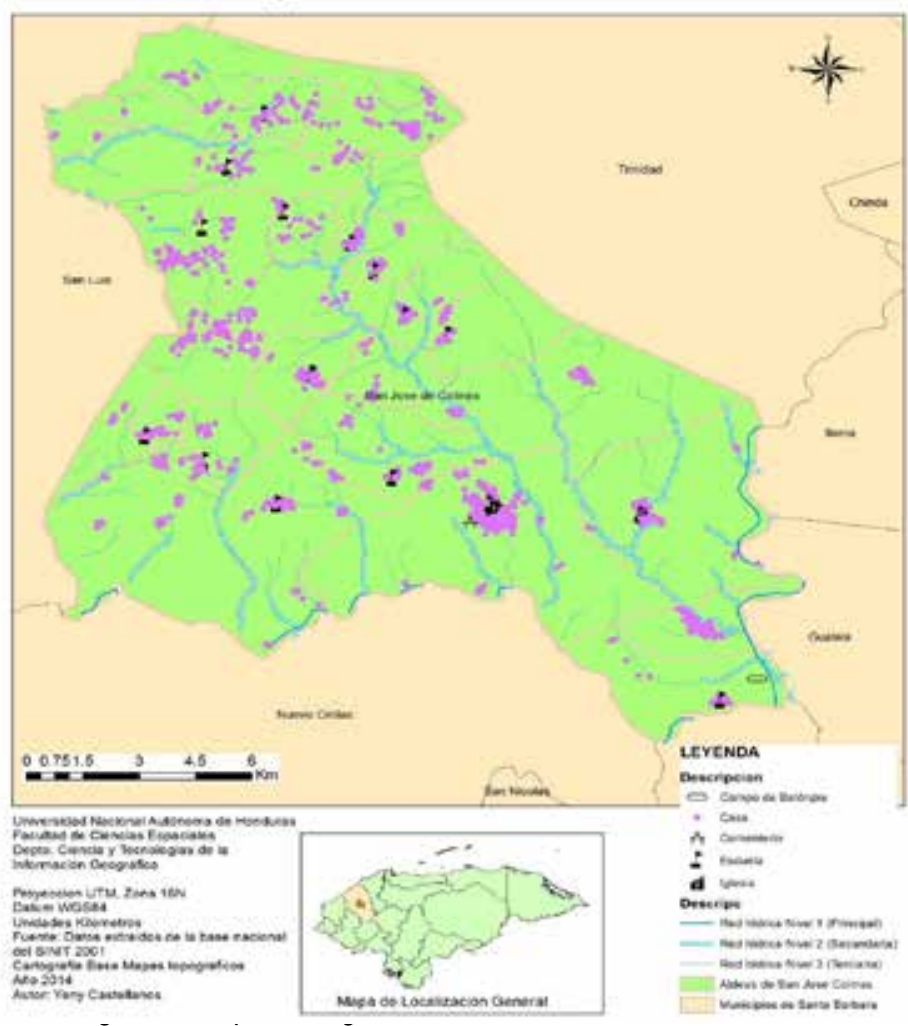

Podemos observar los Tipos de suelos que posee el Municipio de San José Colinas, en donde cabe resaltar que existen numerosos sistemas de clasificación, el que destaca en la data del SINIT del 2001 es la clasificación de Thorp, Baldwin y Kellog (AEPECT, 2001) en donde podemos observar en el mapa de Tipos de Suelo (Figura 3) que en el Municipio de San José Colinas posee suelos Azonales y Zonales.

- Ya se encuentra tabulado un $60 \%$ de los datos proporcionado por la Dirección de Vinculación Universidad Sociedad, el programa utilizado para la tabulación de datos fue el de Microsoft Excel. (Anexo 2). 


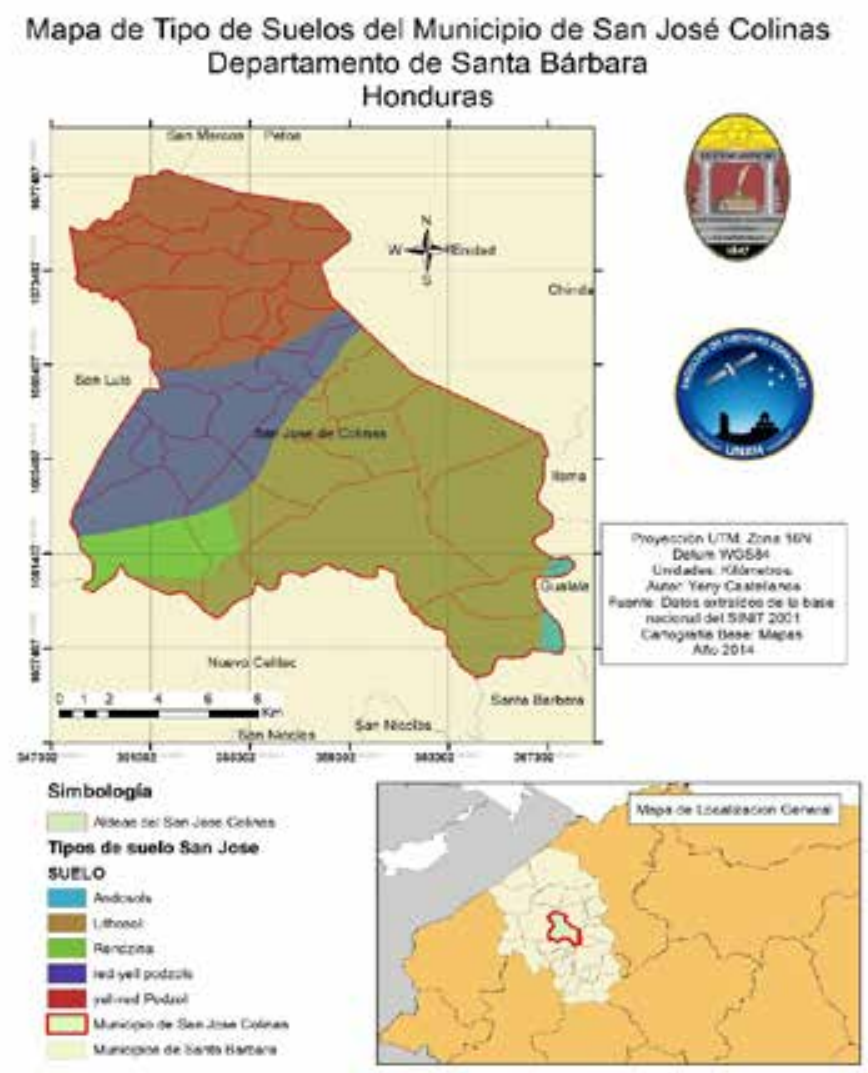

\section{Discusión y Conclusiones}

Al analizar la información que se ha obtenido refleja el desconocimiento de los responsables de recopilar los datos en el campo, al mismo tiempo la importancia que tiene que los datos geoespaciales que sean confiables, ya que son una fuente de información primaria. A su vez la exigencia en la captación de la información geoespacial, que posee una característica única para poder llevar a cabo un análisis espacial real en donde se pueda distinguir las principales causas de las situaciones que no llevan a la prestación correcta de los servicios básicos de la población.

Se requiere la voluntad de las autoridades locales para el acceso de la información, así como de las instancias que colaboran en el proceso de desarrollo de proyectos locales y la concientización de la población y la participación activa de todos los interesados del desarrollo local. 


\section{Bibliografía}

- AEPECT. (27 de agosto de 2001). Seminario Permanente de la Tierra y del Medio Ambiente. Obtenido de http://platea.pntic.mec.es/ cmarti3/CTMA/SUELO/ clasif1.htm

- Buzai, G., Moreno, A., \& Baxendale, C. (2008). Análisis exploratorio de datos espaciales educativos: Aplicación a la Ciudad de Lujan. En C. Baxendale, L. Bevilaqua, G. Buzai, A. Moreno, A. Semorile, \& D. Valdez A., Análisis y Planificación de Servicios Colectivos con Sistemas de Información Geográfica (pág. 149). Madrid: Grafiprint S.L. .

- Gonzalez, M. S. (4,5 y 6 de Octubre de 2011). Foro Internacional MEDAMERICA 2011. Recuperado el septiembre de 2013, de Foro MEDAMERICA: http:// www.ub.edu/medame/foro_ptdr/presentaciones_11.htm

- Hein, A. (Junio de 2009). Fundación Paz Ciudadana. Recuperado el 2013, de La Georreferenciación como herramienta para el diagnóstico de problemas de seguridad ciudadana en el ámbito local: http://www.pazciudadana.cl/docs/ pub_20090623122857.pdf

- Moreno, A. (2008). Los servicios colectivos y el desarrollo territorial. En G. Buzai, A. Moreno, \& C. Baxendale, Análisis y Planificación de Servicios Colectivos con Sistemas de Información Geográfica (pág. 149). Madrid: Grafiprint S. L.

- Ramírez, M. L. (2003). Cálculo de medidas de accesibilidad geográfica, temporal y económica generadas mediante sistemas de información geográfica. Primer Congreso de la Ciencia Cartográfica. 


\section{Anexos}

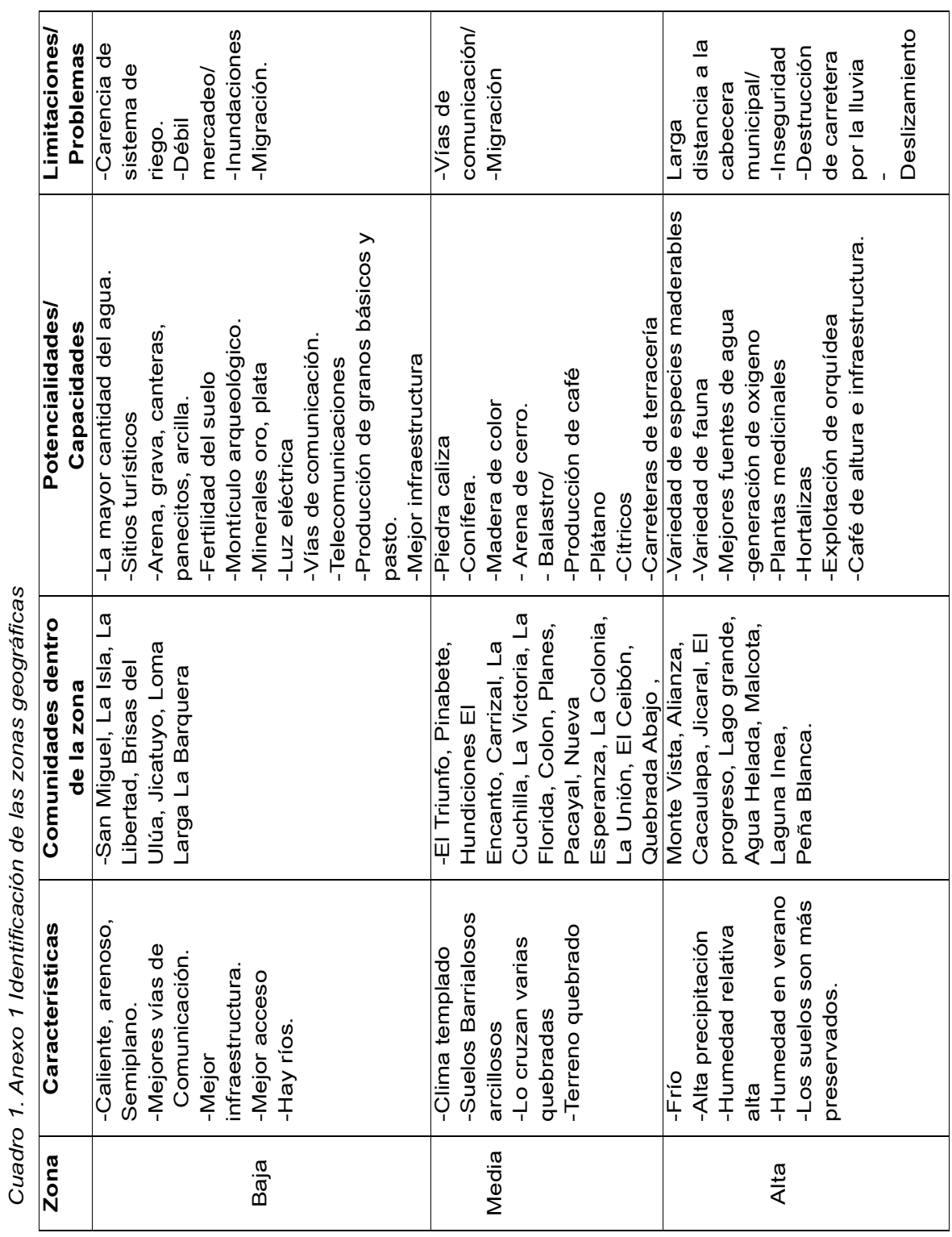

Cuadro 1. Anexo 1 Identificación de las zonas geográficas 


\begin{tabular}{|c|c|c|c|c|c|c|c|c|c|c|}
\hline$d$ & A & in & 5 & D & E & I & 1 & 1 & K & $\Rightarrow$ \\
\hline 1 & na REGION & NOMERE REGION & \begin{tabular}{|c|} 
CODAGO \\
DCPARTAMMINTO
\end{tabular} & DEPARTAMENTO & $\begin{array}{c}\text { COOIGo } \\
\text { Municipto }\end{array}$ & MUNECaPHO & $\begin{array}{l}\text { CODIGO } \\
\text { ADDA }\end{array}$ & ALDEA & $\begin{array}{l}\text { COONGO } \\
\text { CAstuno }\end{array}$ & \\
\hline 573 & 16 & SANTA BARBARA & 15 & SAVIA SÁBEARA & 2602 & ALADA & 160201 & ARADA & 160201012 & $2 \mathrm{TE}$ \\
\hline 534 & 16 & SANTA BARBARA & 15 & SANTra Eó́Jeara & 1602 & ARAOA & 160201 & ARADA & 160201005 & 5 JIN \\
\hline $5 \pi$ & 16 & SANTA BARBARA & 16 & SANTA BARARA & 1602 & ARADA & 160203 & CANOELARIA & 160203002 & $2 \mathrm{CAI}$ \\
\hline 576 & 16 & SANTA BARBARA & 16 & SANTA 8AREARA & 1602 & ARADA & 160203 & CANOLLASLA & 160203020 & D.: \\
\hline$s \pi$ & 16 & SANTA BARBARA & 16 & SANTA BAABAARA & 1602 & ARADA & 160203 & CANOCLAEIA & 160204006 & 590 \\
\hline 578 & 16 & SANTA BABBARA & 16 & SANTA BÁRBARA & 1602 & ARADA: & 160204 & CAULOTALS & 160204001 & $\frac{10}{0}$ \\
\hline $57 y$ & 16 & SANTA BARBARA & 16 & SANTA BÁREARA & 1602 & ARADA & 160205 & FL OCOTAL & & \\
\hline 550 & 16 & SANTA BARBARA & 16 & SANTA EGREALA & 1602 & ARMDS & 160206 & ELL OCOTLLO & 160206002 & $2 \mathrm{EI}$ \\
\hline 581 & 16 & SANTA BARBARA & 16 & SANTA EÁREARA & 1602 & AlAOS & 160207 & EL PALMO & 160207006 & $6 \mathrm{MA}$ \\
\hline 582 & 16 & SANTA BARBARA & 16 & SANTA EAREBARA & 1602 & ARADA & 160207 & EL PALMO & 160207004 & 4 LA \\
\hline 348 & & & & & & & 160207 & EL PALMO & 160207003 & $3<4$ \\
\hline $5 S 4$ & 16 & SANTA BARBAQA & 16 & SANTA RADEARA & 1602 & ARADA & 160208 & EL TULAR & & \\
\hline 565 & 16 & SANTA BARBARA & 15 & SANTA BAREARA & 1602 & ARADA & 160200 & LAS Ekisas of OAO & 160200000 & nit \\
\hline 366 & - T tabia & DIKGRADA HES? & $\operatorname{leg}_{2} 3$ & & & 74 & 160209 & LAS EKISAS DE ORO & 160000007 & $750=$ \\
\hline
\end{tabular}

\section{Cuadro 2. Tabla de tabulación de Datos}

\begin{tabular}{|c|c|c|c|c|c|c|c|c|c|}
\hline BO. REGLON & NOMBRE REGIÓN & \begin{tabular}{|c|} 
CoOtGo \\
DrpaRTAMANTO
\end{tabular} & DEPARTAMENTO & {$\left[\begin{array}{c}\text { CODIGO } \\
\text { MUNICIPIO }\end{array}\right.$} & Municipeso & $\begin{array}{l}\text { COOIGO } \\
\text { AIDIA }\end{array}$ & ALDEA & $\begin{array}{l}\text { CoDo6o } \\
\text { casriuo }\end{array}$ & \\
\hline 16 & SANTA BASBABA & 16 & SANTA NAERARA & 1602 & ARADA & \multicolumn{2}{|c|}{160211 SOACA } & & \\
\hline 15 & SANTA BAGBARA & 16 & SARTA BAERARA & 1603 & AnMA & \multicolumn{2}{|c|}{160301 ATIMA } & 160301008 & 8 LA: \\
\hline 16 & SANTA BAQBARA & 16 & SANTA DAFBARA & 1603 & ATIMA & \multicolumn{2}{|c|}{ 160301 AfIMA } & 160301009 & $9 \cos$ \\
\hline 16 & SANTA BARBARA & 16 & SANTA RAERARA & 1603 & ATIMA & \multicolumn{2}{|c|}{160307 SAN RAFAEL } & 160307004 & $4 \mathrm{ELI}$ \\
\hline 16. & SANTA BARBARA & 16 & SANTA AGERARA & 1603 & ATIMA & \multicolumn{2}{|c|}{160307 SAN RAFAE } & 160307002 & $2 \mathrm{EL}$ \\
\hline 16 & SANTA BARBARA & 16 & SANTA nLERAARA & 1600 & ATIMA & \multicolumn{2}{|c|}{160348 TALANGA } & 160308004 & 4 E: \\
\hline 16 & SANTA BAQBAQA & 16 & SANTA EKBLARA & 1603 & ATMA & \multirow{2}{*}{\multicolumn{2}{|c|}{$\begin{array}{l}160308 \text { TALANGA } \\
160305 \text { ?AN NOE, VE OUENA }\end{array}$}} & 160308006 & 6 LAC \\
\hline 16 & SANTA BARBARA & 26 & SANTA BÁELARA & 2603 & AnMa & & & 160305003 & 3 e. \\
\hline 16 & SANTA BARBARA & 16 & SANTA MARMRA & 1603 & ATMA & \multicolumn{2}{|c|}{$\begin{array}{l}\text { SAN IOSE, DE BUENA } \\
\text { VISTA }\end{array}$} & 160305001 & 1 SAI \\
\hline 16 & SANTA BABBARA & 16 & SAMTA DAERARA & 1601 & AnMA & \multicolumn{2}{|c|}{ 160306 SAN PECATTO } & 160305003 & $3 \mathrm{E}^{\circ}$ \\
\hline 16 & SANTA BARBARA & 16 & SANTA BGs:BARA & 1603 & AnMa & \multicolumn{2}{|c|}{160306 SAN PEOATO } & 160308009 & 9 AiC: \\
\hline 16 & SANTA BABBARA & 16 & SANTA DAERARA & 1603 & ATIMA & 160306 & SAN PEOATTO & 160305009 & $9 \mathrm{SAI}$ \\
\hline 16 & SANTA BARBARA & 16 & SANTA nAEMARA & $160\}$ & lanma & \multirow{2}{*}{160303} & IEMPA & 160303002 & $2 \mathrm{EL} / \mathrm{O}$ \\
\hline - MI TABLA & IMIEGRADA MIV? & 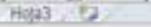 & & & 14 & & $=$ & & , \\
\hline
\end{tabular}

Cuadro 3. Tabla de datos tabulados 